\title{
Applications of Electron Spin Resonance Spectrometry for Reactive Oxygen Species and Reactive Nitrogen Species Research
}

\author{
Masahiro Kohno* \\ New Industry Creation Hatchery Center, Tohoku University, 6-6-10 Aoba, Aramaki, Aoba-ku, \\ Sendai 980-8579, Japan
}

Received 28 January, 2010; Accepted 1 March, 2010; Published online 18 June, 2010

\begin{abstract}
Summary Electron spin resonance (ESR) spectroscopy has been widely applied in the research of biological free radicals for quantitative and qualitative analyses of reactive oxygen species (ROS) and reactive nitrogen species (RNS). The ESR spin-trapping method was developed in the early 1970 s and enabled the analysis of short-lived free radicals. This method is now widely used as one of the most powerful tools for free radical studies. In this report, some of the studies that applied ESR for the measurement of ROS and RNS during oxidative stress are discussed.
\end{abstract}

Key Words: electron spin resonance, chemiluminescence, reactive oxygen species, reactive nitrogen species

\section{Introduction}

Aerobic organisms consume a large amount of oxygen to maintain cellular processes. As a result, reactive oxygen species (ROS) and reactive nitrogen species (RNS) are generated from oxygen $\left(\mathrm{O}_{2}\right)$ utilization. These molecules are assumed to play a role as biological mediators for homeostasis. In turn, homeostasis is maintained by the activity of enzymes such as superoxide dismutase (SOD), catalase, glutathione reductase and glutathione oxidase, all of which control the generation of ROS and RNS in vivo. However, ROS and RNS may cause tissue damage, leading to myocardial infarction and cerebral infarction, for example, and play a pivotal role in preventing microbial infection.

\footnotetext{
*To whom correspondence should be addressed.

Tel: +81-22-795-3976 Fax: +81-22-795-4110

E-mail: mkohno@niche.tohoku.ac.jp

He received "SFRR Japan Award" in 2009 in recognition of his outstanding work.

\section{Biography}

Dr. Kohno is a full professor at New Industry Creation Hatchery Center (NICHe) of Tohoku University. Recently, his main research interests are bio-marker with regard to human injury such as cancer,
}

Therefore, ROS and RNS have been the focus for many lifescience researchers [1-5]. The most common ROS include superoxide anion $\left(\mathrm{O}_{2}{ }^{-}\right)$, hydrogen peroxide $\left(\mathrm{H}_{2} \mathrm{O}_{2}\right)$, hydroxyl radical $\left(\mathrm{HO}^{*}\right)$ and singlet oxygen $\left({ }^{1} \mathrm{O}_{2}\right)$, all of which are more reactive than $\mathrm{O}_{2}$. Of these, $\mathrm{H}_{2} \mathrm{O}_{2}$ is a comparatively stable molecule and is a key substrate for enzymes such as $\mathrm{P} 450$ peroxidase, and plays a role in $\mathrm{O}_{2}$ storage. By contrast, unlike $\mathrm{O}_{2}{ }^{--}, \mathrm{H}_{2} \mathrm{O}_{2}$ and $\mathrm{HO}$, the characteristics of ${ }^{1} \mathrm{O}_{2}$ are not well known because of its high reactivity and the lack of techniques to stabilize this molecule. The most common RNS are nitrogen monoxide (NO), nitrogen dioxide $\left(\mathrm{NO}_{2}\right)$ and nitrogen oxide $\left(\mathrm{N}_{2} \mathrm{O}_{4}\right)$. The term "free radical" is often confused with ROS and RNS. Free radicals are atoms or molecules containing an unpaired electron, such as $\mathrm{O}_{2}, \mathrm{O}_{2}{ }^{--}$, $\mathrm{HO}^{*}, \mathrm{NO}^{*}$ and $\mathrm{NO}_{2}{ }^{\circ}$. Under normal conditions (e.g., temper-

$\overline{\text { diabetes, kidney disease and allergy. To resolve these problems, he }}$ applied new analytical methods for measuring free radicals called reactive oxygen species by using electron spin resonance and chemical luminescence devices. Recently he has successfully proposed $\mathrm{HOOOH}$ as a new reactive oxygen species. This finding gives one of the most important interpretations explaining homoeostasis in vivo in relation to the function of superoxide dismutase. He was awarded the Society for Free Radical Research Japan Award in 2009. 
(a)

\section{Generation system of ROS}

Xanthine-oxidase

NADPH-oxidase

NADPH dehydrogenase

Ubiqinol-Cyt.c reductase

NADPH-Cyt.P450 reductase

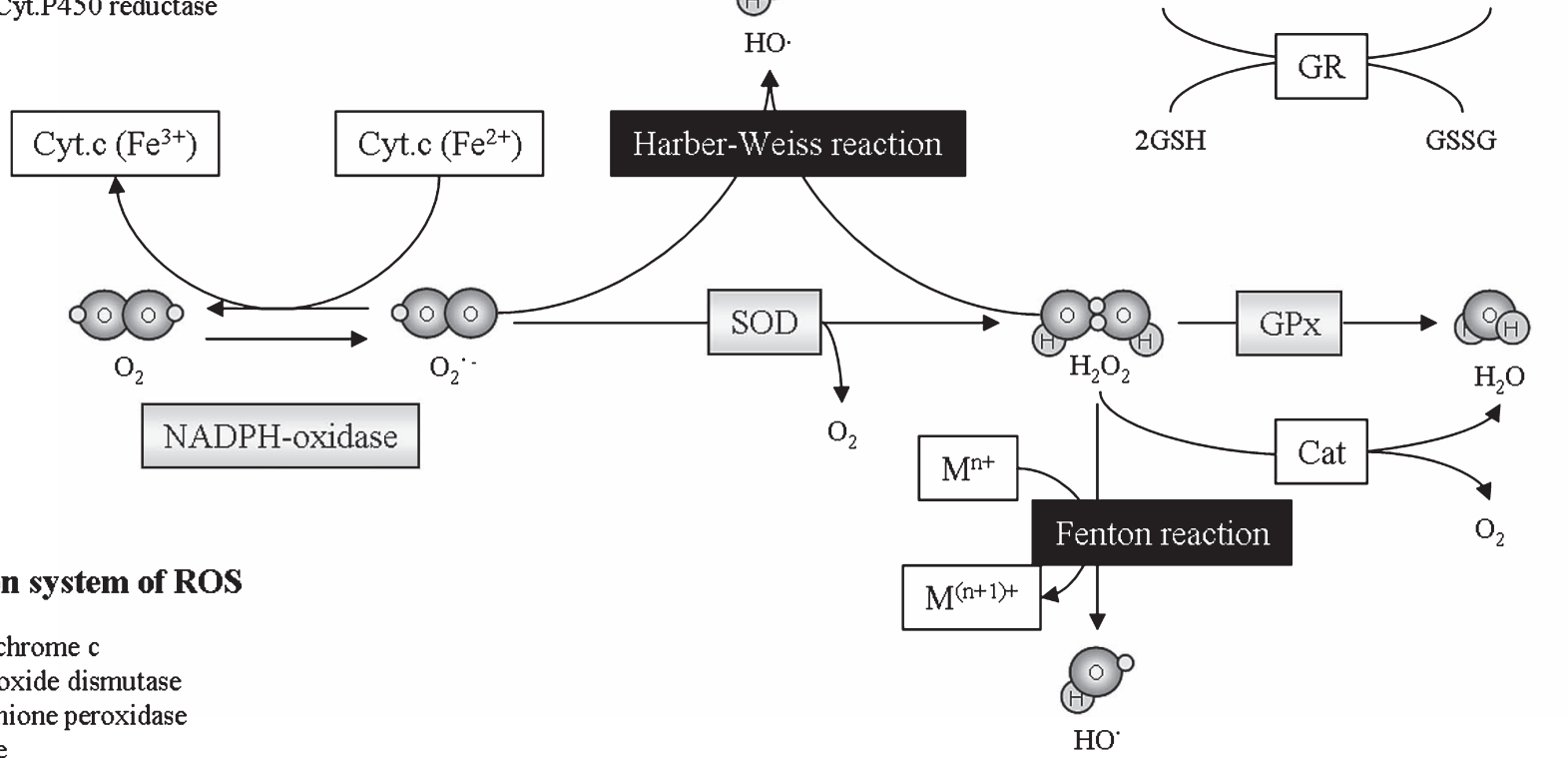

\section{Reduction system of ROS}

Cyt.c: cytochrome c

SOD:superoxide dismutase

GPx:glutathione peroxidase

Cat:catalase

$\mathrm{HO}^{\circ}$

GR:glutathione reductase

GSH:reduced form glutathione

GSSG:oxidatized form glutathione

NADPH: nicotinamide adenine dinucleotide phosphate

(b)

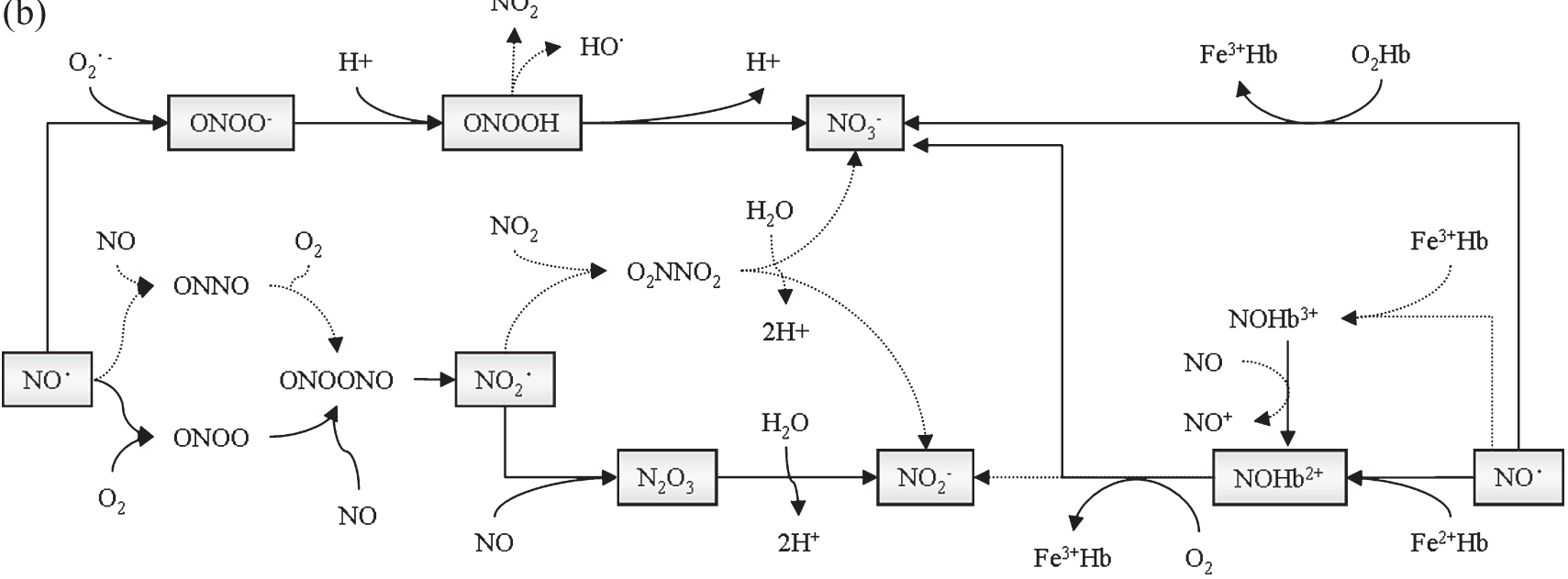

Fig. 1. (a) Schematic illustration summarizing the biological systems involved in the generation and decomposition of ROS.

(b) Schematic illustration summarizing the chemical reactions involved in the generation and decomposition of RNS.

ature and pressure), free radicals are usually unstable because of an unpaired electron, which leads to their high reactivity.

As shown in Figs. 1a and 1b, aerobic organisms have endogenous systems to produce and eliminate ROS and
RNS to maintain homeostasis. Because the mechanisms involved in the production and regulation of ROS and RNS in vivo are complex, most studies have been conducted in vitro. Researchers have attempted to clarify the causal relationship between tissue damage and ROS or RNS levels. 
However, because ROS generated in the living body cannot be qualitatively analyzed, the outcomes of many studies have resulted in a deduction or an assumption rather than definitive evidence. Therefore, qualitative and quantitative analytical methods are needed to assess the generation of ROS and RNS in vivo, and we need to elucidate the biological effects of ROS and RNS. Spectroscopic instruments that detect fluorescence, phosphorescence and chemiluminescence have been used for the analysis of ROS and RNS. However, a question arises if these analytical methods also measure nonspecific coloring or radiation. For example, it is difficult to directly measure ROS generated by the electron transport system in mitochondria, the NADPH oxidation enzyme system in leukocytes, the xanthine oxidation enzyme system in the cytoplasm, and the p450 oxidation enzyme system in liver tissue. Superoxide dismutase (SOD) is one of the most widely used enzymes to analyze ROS generation. SOD, which is expressed in the liver, brain and heart tissues, is related to the maximum life span potential and anti-inflammatory pathways. Another widely used molecule is NO, which is a key mediator for relaxation of vascular smooth muscle, and is generated by two enzymes, inducible nitric oxide synthase (i-NOS) and constitutive nitric oxide synthase (c-NOS). A number of methods are available to measure NO, including the Griess method, the electrode method, the chemical luminescence method, and the ozone chemical luminescence method. However, poor selectivity, accuracy and methodology have been reported to be limitations of these methods to measure RNS.

In contrast, the ESR technique coupled with the spinoxidation method, in which 2-(4-carboxyphenyl)-4,4,5,5tetramethyl-imidazoline-1-oxyl 3-oxide (carboxyl-PTIO) is used to measure NO, was first proposed by Akaike in 1993 [6]. This method offers greater accuracy than the earlier methods to measure NO.

\section{Development of ESR Methods}

The ESR phenomenon was first discovered in 1945, and ESR-based methods have since become widely used because the process can detect free radicals without interference from the sample properties, including its phase (solid, liquid or gas). Therefore, if the ESR spectrum can be observed using an ESR instrument, we can identify the free radical present in a sample. Since free radicals contain an unpaired electron, they are very reactive and generally unstable at room temperature.

The ESR spin-trapping method is a well known, useful tool to detect short-lived radicals such as ROS and RNS. A five-membered ring nitron derivative, 5,5-dimethyl-1pyrroline- $N$-oxide (DMPO), is widely considered to be the most useful and powerful spin-trapping reagent.
The spin-trapping method was originally proposed by Janzen et al. and several other researchers in the late $1960 \mathrm{~s}$ [7-10]. The spin label method was reported by Ohnishi et al. in 1965 [11], and the reagents for spin labeling are used for ESR imaging. Fundamentally, spin-trapping reagents react with short-lived radicals, which are subsequently changed to long-lived radicals called spin-adducts. By observing the ESR spectra of spin-adducts, the ESR characteristics can be obtained, including the g-value, hyperfine coupling constant (hfcc) and spin concentration.

As shown in Fig. 2, two types of spin-trapping compounds, nitrone and nitroso compounds, can react with free radicals $\left(\mathrm{R}^{*}\right)$ and produce a stable amino oxide radical. Thus, two types of reagents have been used for the spintrapping method. On the other hand, the spin-labeling method can be used to measure the dynamic characteristics of molecules combined with unsaturated fatty acids, phospholipids or cholesterol, and this method has been applied for ESR imaging.

A spectrum is analyzed in terms of its hfcc and g-values. The g-value is determined from the resonance magnetic field and the resonance frequency observed as ESR signals. On the other hand, hfcc represents the splitting of an ESR spectrum, and is determined by analyzing the splitting of the spectrum of spin adduct [12]. The concentration of a free radical is obtained as the double integrals of the ESR spectrum of a spin adduct (i.e., the area of the spectrum). As described above, the ESR spin-trapping method now allows us to qualitatively and quantitatively analyze ROS and RNS.

\section{Application of ESR to Measure Oxygen Free Radicals}

The nitrone compound used for ESR spin-trapping can be used to detect (and measure) oxygen free radicals such as $\mathrm{O}_{2}{ }^{--}$and $\mathrm{HO}^{*}$. Meanwhile, nitroso compounds are mainly used to detect carbon radicals. Desirable characteristics of spin-trapping reagents include high solubility and stability in water, and the ability to interact with free radicals. Furthermore, high reactivity is required to observe the formation of free radicals, while low reactivity is needed to measure elimination activity. It is also desirable that the resulting spin adduct is stable for a relatively a long time and that the spectrum can be easily analyzed.

In our initial studies in 1985, we used DMPO, a high quality agent, as the spin-trapping agent [13], and was supplied from domestic chemical companies. We found that early batches of DMPO imported from other countries had low purity and reacted with blood and living tissues, Moreover, carbon-based radicals derived from the reagent were observed. Since then, domestically produced DMPO has been widely used.

Since 1976, many researchers have used DMPO for in vitro studies of living cells, particularly neutrophils. Notably, 


\section{(a) Nitroso compound}<smiles>[R]N([O])C(C)(C)C</smiles>

2-Methyl-2-nitroso-propane

(MNP)

\section{(b) Nitrone compound}<smiles>CC(C)(C)[N+]([O-])=Cc1ccccc1</smiles>

$\alpha$-Phenyl- $N$-t-butylnitrone

(PBN)
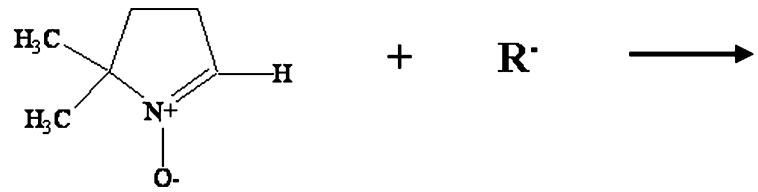

5,5-Dimethyl-1-pyrroline- $N$-oxide (DMPO)
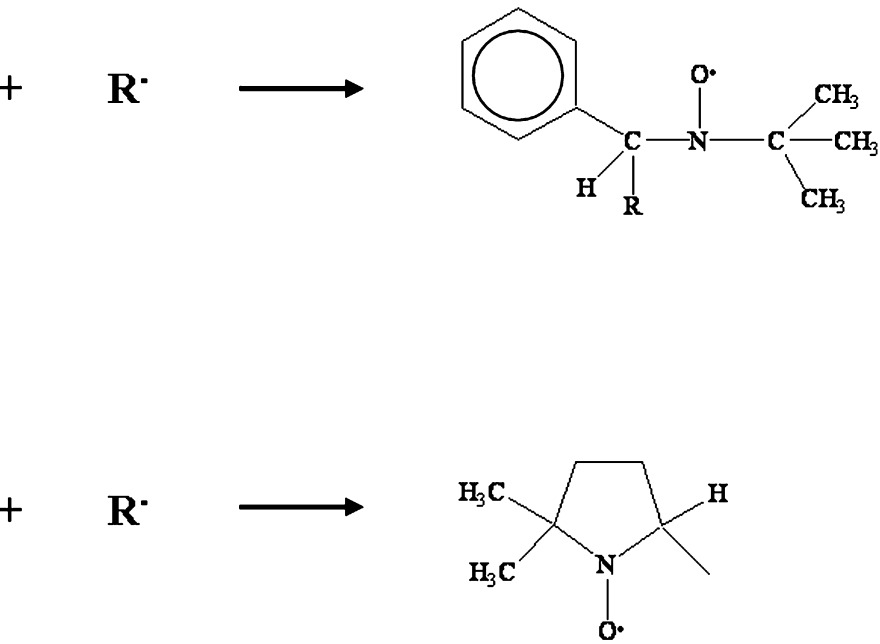

Fig. 2. Chemical structures of spin-trapping agents, and the reactions between the agents and a free radical to form spin adducts.

DMPO is easy to handle for 'beginners', is relatively cheap and is a stable reagent. The main advantage of DMPO is that it is easy to analyze ESR spectra. However, DMPO is cytotoxic at high concentrations, limiting its use. Thus, other spin-trapping agents with low toxicity have been identified and can be used for in vitro studies of living cells and bacteria [14-17].

ESR spin-trapping methods have also been applied to measure SOD and SOD-like activities. The typical enzyme reactions for the generation of $\mathrm{O}_{2}{ }^{--}$are shown in Fig. 3, and both reaction systems have been used to evaluate the superoxide scavenging activity of many natural products [13, 18-29]. These methods also can be applied for kinetic analyses [30, 31]. For example, the DMPO spin-trapping method has been used to determine the enzyme reaction velocity of xanthine oxidizing enzyme (XOD) and an NADPH oxidizing enzyme [18, 32].

The production of $\mathrm{HO}^{*}$ has been used to determine the velocity constant of the reaction between an iron ion with $\mathrm{H}_{2} \mathrm{O}_{2}$. Similarly, other studies have quantitatively analyzed the rate of $\mathrm{HO}^{*}$ generation from water exposed to ultrasonic irradiation [33-35].

The ESR spin-trapping method can be applied to measure the superoxide scavenging ability and hydroxyl radical scavenging ability of anti-inflammatory agents, anti-oxidants and Chinese herbal medicines. Recently, we have demonstrated the production of a new ROS, HOOOH, through xanthine oxidase- and NADPH oxidase-mediated reactions between a $\mathrm{O}_{2}{ }^{--}$and $\mathrm{HO}^{\bullet}[36,37]$.

\section{Application of ESR to Measure Nitrogen Oxide (NO)}

NO is well established as a vasodilatory mediator; thus, accurate and reliable methods to measure NO levels are essential. As shown in Fig. 4, NO is generated by NO synthase in vivo, with L-arginine as a substrate.

The use of ESR spectrometry to measure NO was first reported in 1993 [6]. As shown in Fig. 5, a stable free radical, carboxyl-PTIO reacts with NO, and carboxyl-PTI is formed. The Griess, electrode and chemiluminescence 
<smiles>O=C1NC(=O)C2N=CNC2N1</smiles>

Xanthine

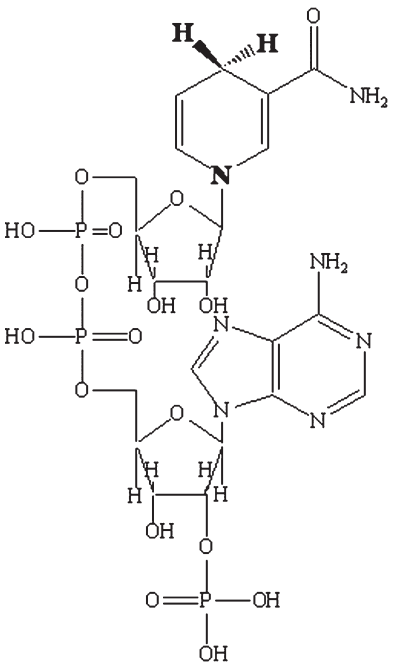

$\mathrm{NADPH}$

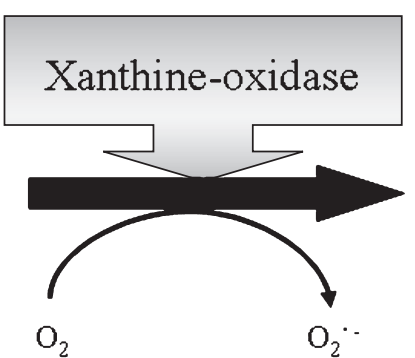<smiles>O=C1NC(=O)C2NC(=O)NC2N1</smiles>

Uric acid

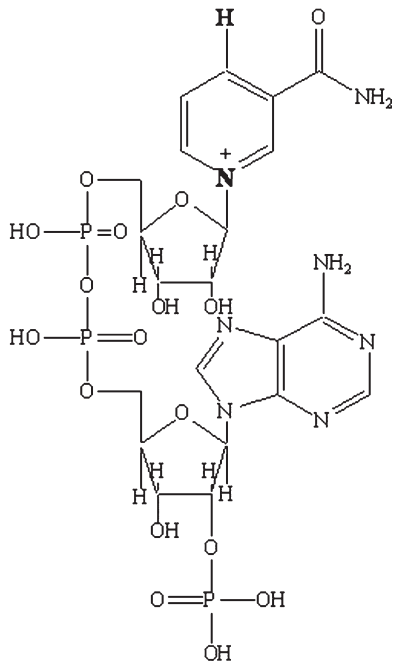

$\mathrm{NADP}^{+}$

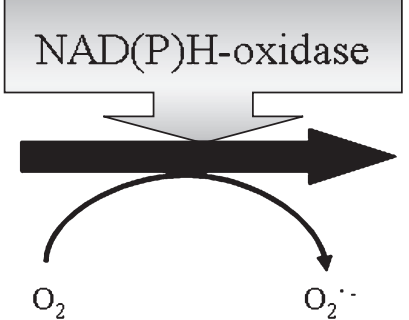

$\mathrm{O}_{2} \cdot$

Fig. 3. Xanthine oxidase and NADPH oxidase: representative enzymes that contribute to the production of $\mathrm{O}_{2}^{--}$in biological systems.<smiles>N=C(N)NCCCC(N)C(=O)O</smiles>

L-arginine
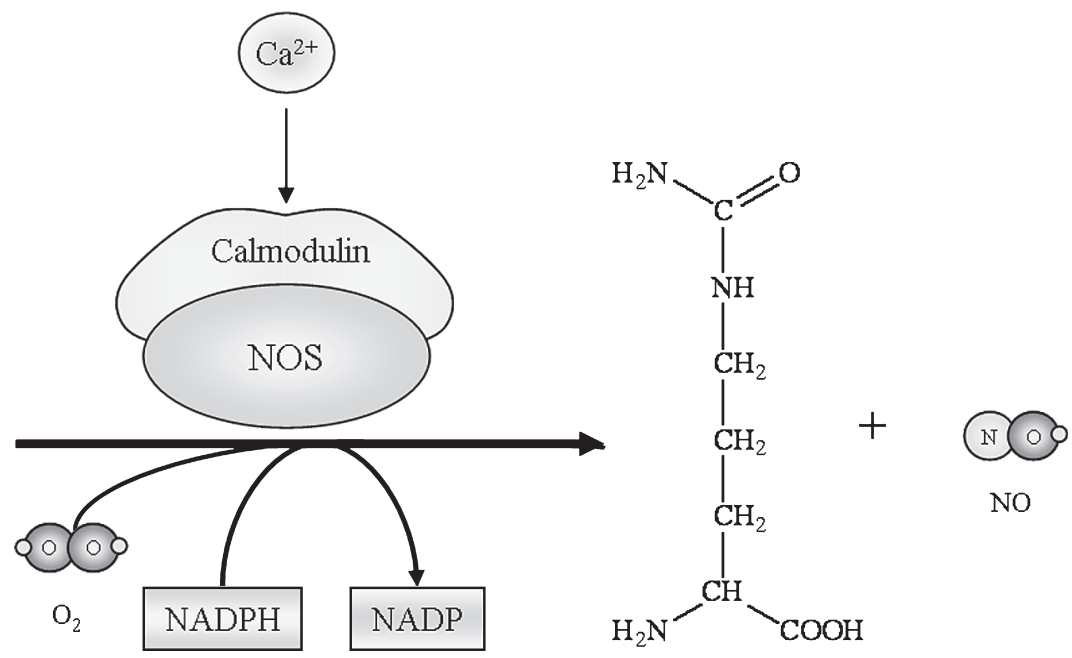

Fig. 4. Schematic illustration for the formation of NO by inducible NO synthase (i-NOS). 


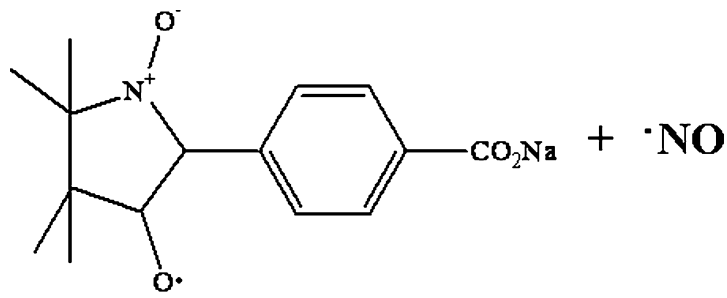

Carboxy-PTIO

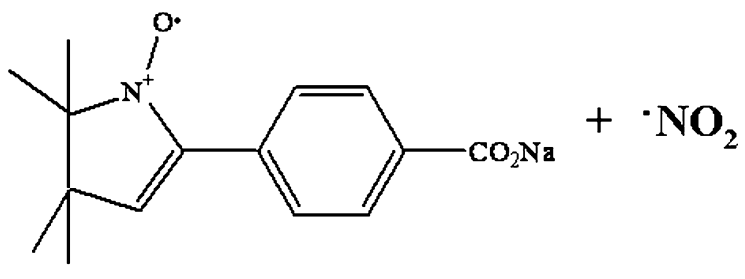

Carboxy-PTI

Fig. 5. Chemical reaction between the stable free radical carboxyl-PTIO and NO, which is used for the quantitative and qualitative assessment of NO.

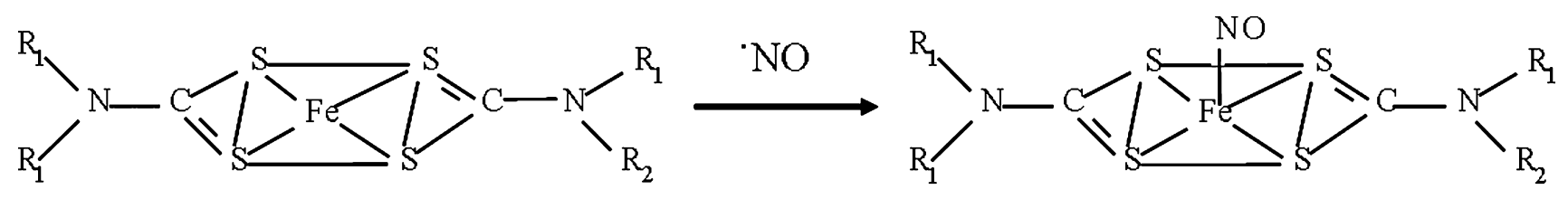

(a)<smiles>CN(CC(O)C(O)C(O)C(O)CO)C(=S)S[NH3+]</smiles>

(b)<smiles></smiles>

(c)

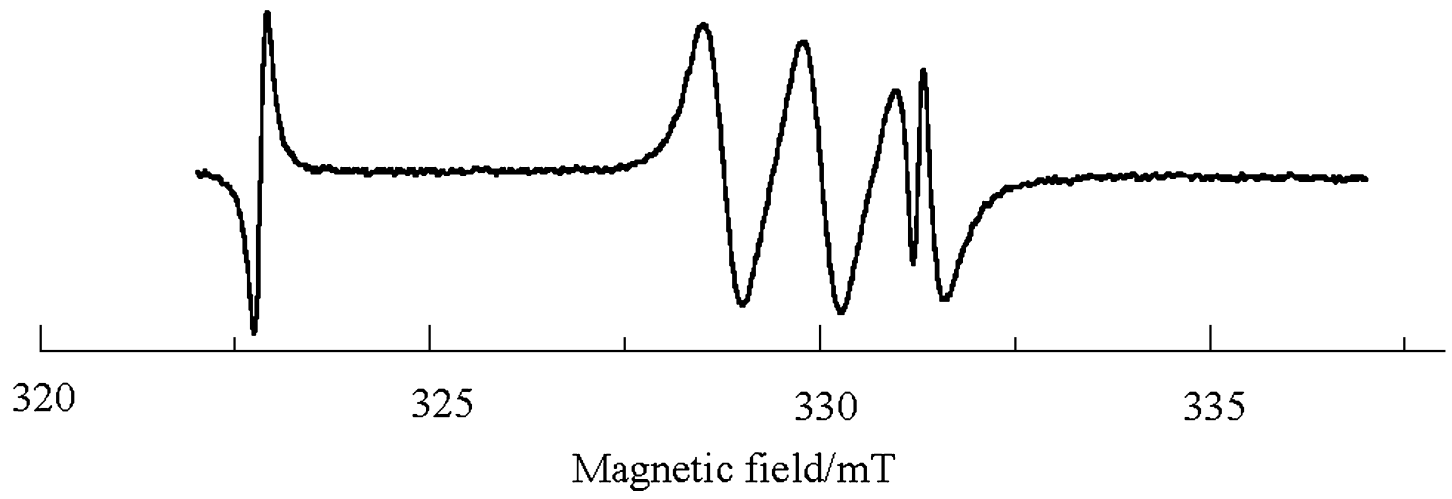

Fig. 6. Chemical reactions between Fe-bisMGD or Fe-bisDTCS, with NO: novel methods for the quantitative and qualitative assessment of NO at room temperature. (a) and (b) show the structures of MGD and DTCS, respectively, and (c) shows a representative ESR spectrum of $\mathrm{MGD}_{2}-\mathrm{Fe}-\mathrm{NO}$. 
methods, for example, are the most widely used conventional methods [38-42]. The PTIO method offers higher selectivity and higher sensitivity compared with these conventional methods.

Before the introduction of the PTIO method, hemoglobin had been used as the spin-trapping agent [43]. This method used ESR to measure the quantity of NO-bound hemoglobin. However, this method is inconvenient because it cannot be conducted at room temperature or in a solution.

More recently, another method, as summarized in Fig. 6, has been developed to measure NO using the reagents MGD [ $N$-(dithiocarbamoyl)- $N$-methyl-D-glucamine, sodium salt] and DTCS [ $N$-(dithiocarboxy)sarcosine, disodium salt, dehydrate], which possess an iron ion [44]. Unlike the NObound hemoglobin method, the MGD/DTCS method can be performed at room temperature and in an aqueous solution.

\section{Advantages and Limitations of the ESR Spin-Trap- ping Method}

Advantages of the ESR-spin-trapping method include the following.

1. Free radicals generated by chemical systems or biological systems can be detected and identified by observing the ESR spectrum of a spin adduct.

2. The characteristics (g-value, hfcc, a-value, alignment, line width, $\Delta \mathrm{W}$ ) of free radicals can be determined by analyzing the ESR spectrum of a spin adduct.

3. The ESR spectrum of a spin adduct can be used for quantitative analysis of free radicals by comparing the peak area with those obtained from stable radicals.

4. ESR spin-trapping can be used for kinetic analyses and determine the formation and elimination velocities of a free radical.

Limitations of the ESR spin-trapping method include the following.

1. If a free radical reacts immediately with a molecule other than the spin-trapping agent, the spin adduct will not be generated, and cannot be detected by ESR.

2. If a spin adduct is present with reducing agent, it may be neutralized.

3. If a spin adduct decomposes, a new spin adduct may be generated.

4. If the hfcc is the only ESR parameter determined for a spin adduct, the electron distribution and the molecular structure of the free radical cannot be determined.

\section{Evaluation of Oxidative Stress by ESR}

In this paper, I have provided an overview of the development of ESR techniques and their application to the biomedical field. DMPO, a conventional spin trapping agent, has been used in many ROS studies. Furthermore, spin- trapping agents with reduced cytotoxicity have also been developed. In addition to chemical and in vitro enzyme reaction systems, these reagents have been used to evaluate in vitro ROS production in living cells such as leukocytes. Nevertheless, questions remain on the biological role of SOD. For example, Sawyer questioned whether oxidative injury is actually caused by $\mathrm{O}_{2}{ }^{--}$, because the oxidative capacity of the $\mathrm{O}_{2-}^{--}$is lower than that of the $\mathrm{HO}^{*}$ [45]. Therefore, it is unclear whether SOD is relevant to the maximum life span potential of humans, as is the case with anti-aging and anti-inflammation. Similarly, it has been demonstrated that the oxidative capacity of $\mathrm{NO}^{*}$ is lower than that of $\mathrm{HO}^{\circ}$. It has been suggested that an active species other than ROS and NOS is present in biological systems [46]. In fact, we recently proposed $\mathrm{HOOOH}$ to be one such candidate molecule, as summarized in Fig. 7 [35].

The speculation arose from chemiluminescence studies, and is similar to the phenomenon reported by Beckman and others, who investigated ozone chemiluminescence [46]. I further speculate that $\mathrm{NO}^{*}$ reacts with $\mathrm{HOOOH}$ to form $\mathrm{HOOO}^{*}$ and $\mathrm{NO}_{2}$. If $\mathrm{HOOO}^{*}$ is a more reactive oxygen species than $\mathrm{HO}^{\prime}$, the anti-oxidative function of SOD can be determined. This will provide evidence for the ability of SOD, which changes a $\mathrm{O}_{2}{ }^{--}$to $\mathrm{H}_{2} \mathrm{O}_{2}$, to inhibit oxidative injury in humans. Fig. 8 shows the traditional concept for oxidative injury associated with SOD activity. Based on this concept, $\mathrm{H}_{2} \mathrm{O}_{2}$ is generated by SOD, resulting in an increase in $\mathrm{HO}^{\circ}$.

$$
\begin{aligned}
& \mathrm{HO}+\mathrm{HOO}^{*} \rightarrow \mathrm{HOOOH} \\
& \mathrm{NO}+\mathrm{HOOOH} \rightarrow \mathrm{HOOO}+\mathrm{NOH}
\end{aligned}
$$

In addition, it has been proposed that SOD suppresses a reaction between $\mathrm{O}_{2}{ }^{--}$and $\mathrm{NO}$, which generates highly reactive peroxynitrate. However, this hypothesis is theoretically contradicted because the reactivity of $\mathrm{NO}_{2}$ is lower than that of ROS.

We have focused on the existence of HOOO; which might cause oxidative injury in vivo. Although the generation of HOOO in vivo has not yet been detected, there is strong evidence for the existence of $\mathrm{HOOOH}$ in vitro (Fig. 9) [47]. This new concept enables us to explain how SOD protects the body from oxidative injury by scavenging $\mathrm{O}_{2}{ }^{-}$ and decreasing the amount of HOOO', which might injure cells and tissues.

The introduction of ESR techniques has already provided significant advances in the understanding of ROS and RNS, but I expect further discoveries by researchers investigating free radicals in the life sciences.

\section{Conclusions}

In this paper, I have reviewed the historical and theoretical 


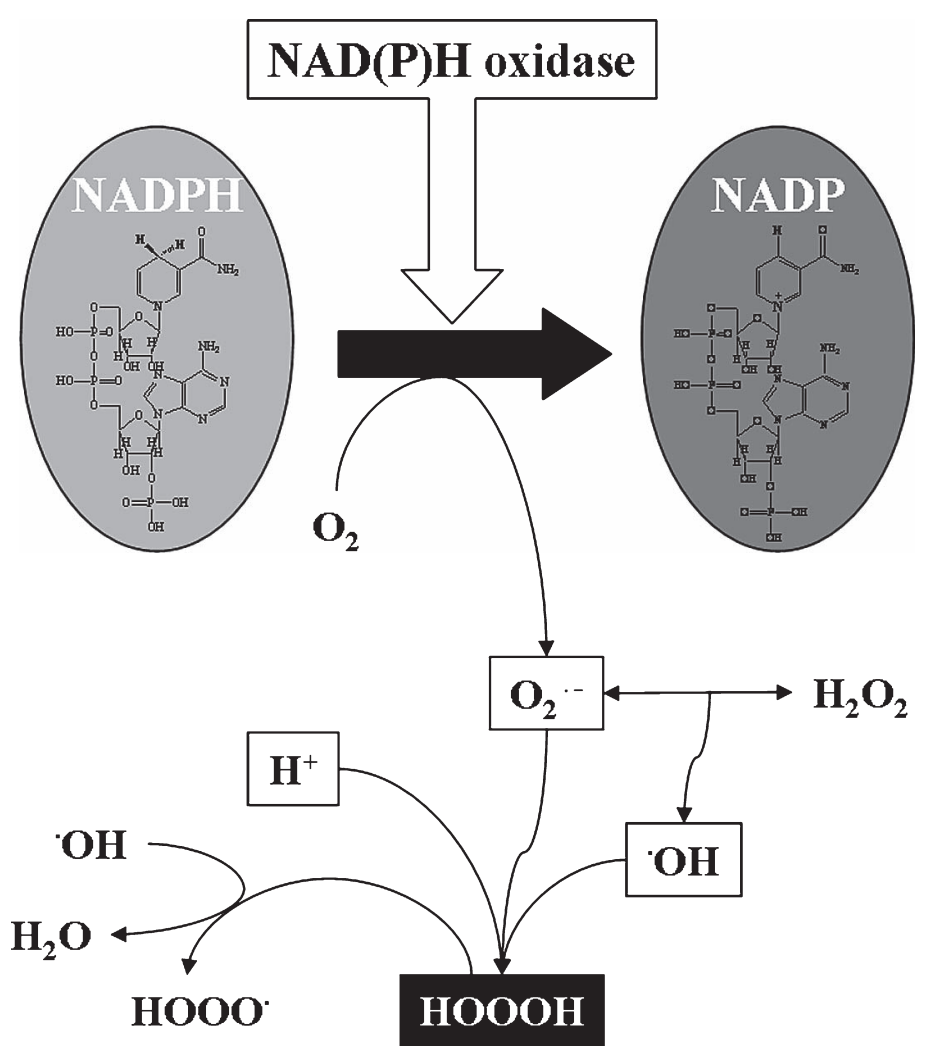

Fig. 7. Proposed mechanism for the formation of $\mathrm{HOOOH}$ via NADPH oxidation.

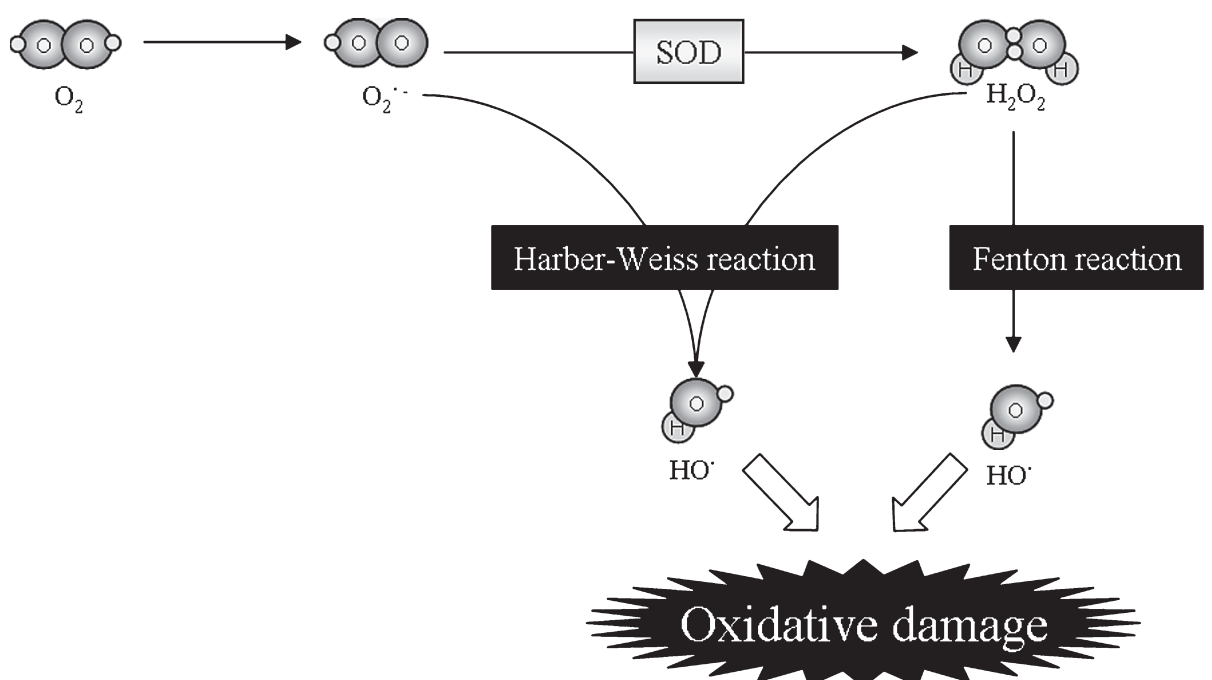

Fig. 8. Schematic illustration for the traditional concept of oxidative injury and the pivotal role of SOD in the generation of ROS.

backgrounds of ESR technology and its application to free radical studies in the life sciences. In addition, the recent progress in this field was discussed. One of the major advances in this technology was the development of highquality spin-trapping agents that react with short-lived radicals and convert them to long-lived radicals called spin-adducts. This has made it possible to quantitatively and qualitatively analyze radicals in vitro. More recently, spin reagents with low cytotoxicity have been developed, allowing the investigation of radical formation at the cellular 


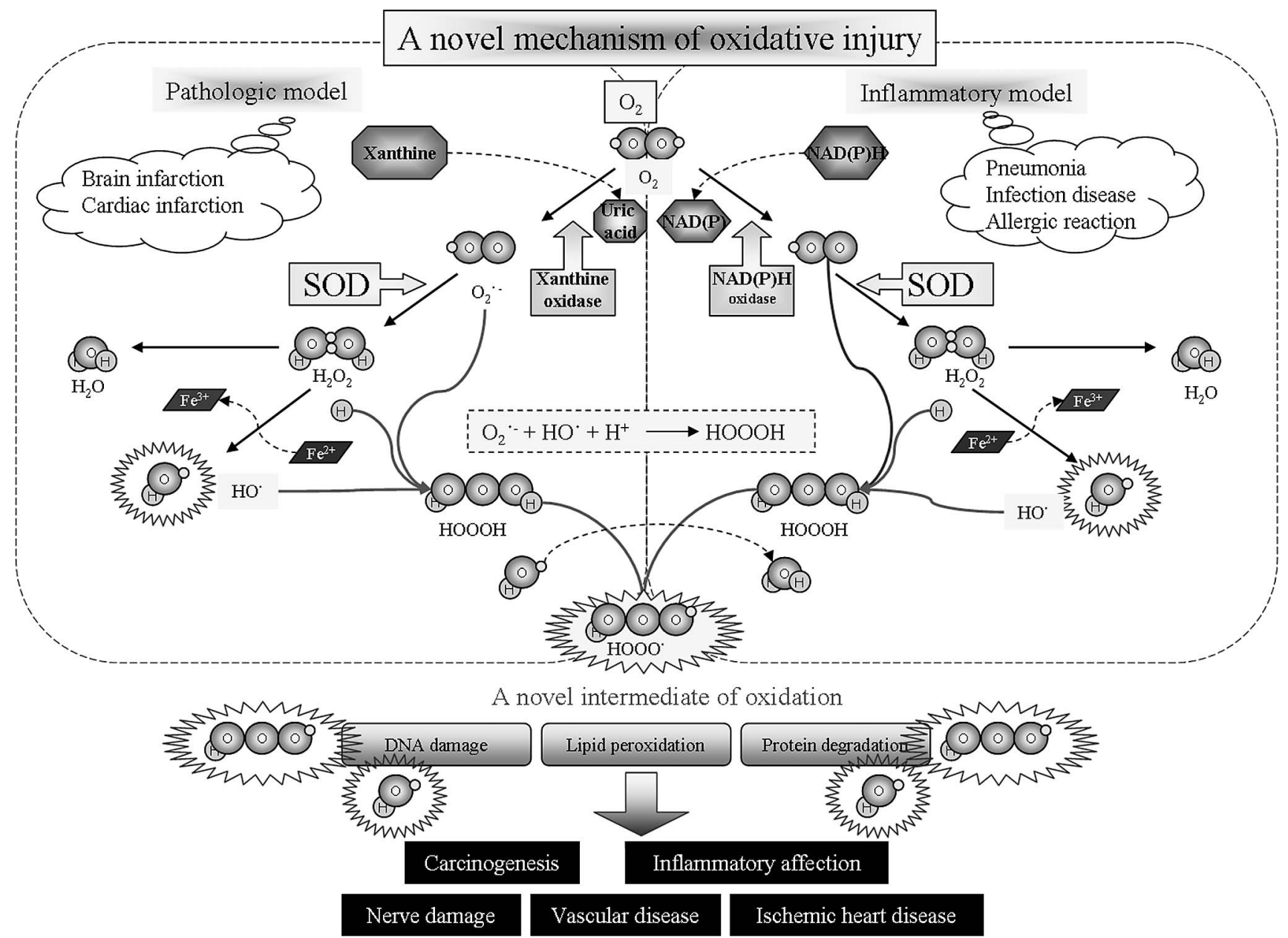

Fig. 9. Schematic illustration of a new concept proposing the pivotal role of a novel oxygen intermediate in oxidative injury.

level. Furthermore, the use of ESR in combination with other technologies can provide valuable novel findings. For example, we have recently postulated the existence of a new oxygen intermediate in biological enzyme systems, which are known to generate ROS, by using ESR in combination with chemiluminescence. I strongly believe that these novel findings have made substantial progress in life sciences research.

\section{Acknowledgments}

I wish to thanks my many colleagues with whom I have discussed this technique, in particular Professor Yoshimi Niwano and Assistant Professor Emiko Sato, New Industry Creation Hatchery Center, Tohoku University. Their contribution to this paper has been very great and I take pleasure in acknowledging the important part played by them.

\section{Abbreviations}

ESR, electron spin resonance; ROS, reactive oxygen species; RNS, reactive nitrogen species; SOD, superoxide dismutase; $\mathrm{O}_{2}{ }^{--}$, superoxide anion; $\mathrm{HO}^{\circ}$, hydroxyl radical; ${ }^{1} \mathrm{O}_{2}$, singlet oxygen; $\mathrm{NO}$, nitrogen monoxide; $\mathrm{NO}_{2}$, nitrogen dioxide; $\mathrm{N}_{2} \mathrm{O}_{4}$, nitrogen oxide; i-NOS, inducible nitric oxide synthase; c-NOS, constitutive nitric oxide synthase; DMPO, 5,5-dimethyl-1-pyrroline- $N$-oxide; hfcc, hyperfine coupling constant, carboxy-PTIO, 2-(4-carboxyphenyl)4,4,5,5-tetramethylimidazoline-1-oxyl-3-oxide; MGD, $N$ (dithiocarbamoyl)- $N$-methyl-D-glucamine (sodium salt); DTCS, $N$-(dithiocarboxy)sarcosine (disodium salt, dehydrated).

\section{References}

[1] McCord, J.M.: Free radicals and inflammation: protection of synovial fluid by superoxide dismutase. Science, 185, 529531, 1974.

[2] Fridovich, I.: The biology of oxygen radicals. Science, 201, 
875-880, 1978.

[3] Tolmasoff, J.M., Ono, T., and Cutler, R.G.: Superoxide dismutase: correlation with life-span and specific metabolic rate in primate species. Proc. Natl. Acad. Sci. U.S.A., 77, 2777-2781, 1980.

[4] Sawyer, D.T. and Valentine, J.S.: How super is superoxide? Acc. Chem. Res., 14, 393-400, 1981.

[5] Palmer, R.M., Ferrige, A.G., and Moncada, S.: Nitric oxide release accounts for the biological activity of endotheliumderived relaxing factor. Nature, 327, 524-526, 1987.

[6] Akaike, T., Yoshida, M., Miyamoto, Y., Sato, K., Kohno, M., Sasamoto, K., Miyazaki, K., Ueda, S., and Maeda, H.: Antagonistic action of imidazolineoxyl $\mathrm{N}$-oxides against endothelium-derived relaxing factor/ ${ }^{\circ} \mathrm{NO}$ through a radical reaction. Biochemistry, 32, 827-832, 1993.

[7] Lagercrantz, C.: Spin trapping of some short-lived radicals by the nitroxide method. J. Phys. Chem., 75, 3466-3475, 1971.

[8] Janzen, E.G.: Spin trapping. Acc. Chem. Res., 4, 31-40, 1971.

[9] Janzen, E.G. and Evans, C.A.: Rate constants for spin trapping tert-butoxy radicals as studied by electron spin resonance. J. Am. Chem. Soc., 95, 8205-8206, 1973.

[10] Harbor, J.R.: An electron spin resonance study of spin adducts of $\mathrm{OH}$ and $\mathrm{HO}_{2}$ radicals with nitrones in the ultraviolet photolysis of aqueous hydrogenperoxide solutions. Can. J. Chem., 52, 3549-3553, 1974.

[11] Ohnishi, S.I. and McConnell, H.M.: Interaction of the radical ion of chlorpromazine with deoxyribonucleic acid. J. Am. Chem. Soc., 87, 2293, 1965.

[12] Buettner, G.R.: Spin trapping: ESR parameters of spin adducts. Free Radic. Biol. Med., 3, 259-303, 1987.

[13] Ueno, I., Kohno, M., Yoshihira, K., and Hirono, I.: Quantitative determination of the superoxide radicals in the xanthine oxidase reaction by measurement of the electron spin resonance signal of the superoxide radical spin adduct of 5,5-dimethyl-1-pyrroline-1-oxide. J. Pharmacobiodyn., 7, 563-569, 1984.

[14] Babior, B.M., Curnutte, J.T., and McMurrich, B.J.: The particulate superoxide-forming system from human neutrophils. Properties of the system and further evidence supporting its participation in the respiratory burst. J. Clin. Invest., 58, 989-996, 1976.

[15] Ueno, I., Kohno, M., Mitsuta, K., Mizuta, Y., and Kanegasaki, S.: Reevaluation of the spin-trapped adduct formed from 5,5dimethyl-1-pyrroline-1-oxide during the respiratory burst in neutrophils. J. Biochem., 105, 905-910, 1989.

[16] Nishizawa, M., Kohno, M., Nishimura, M., Kitagawa, A., and Niwano, Y.: Non-reductive scavenging of 1,1-diphenyl2-picrylhydrazyl (DPPH) by peroxyradical: a useful method for quantitative analysis of peroxyradical. Chem. Pharm. Bull., 53, 714-716, 2005.

[17] Saito, K., Takahashi, M., Kamibayashi, M., Ozawa, T., and Kohno, M.: Comparison of superoxide detection abilities of newly developed spin traps in the living cells. Free Radic. Res., 43, 668-676, 2009.

[18] Mitsuta, K., Mizuta, Y., Kohno, M., Hiramatsu, M., and Mori, A.: The application of ESR spin-trapping technique to the evaluation of SOD like activity of biological substances. Bull. Chem. Soc. Jpn., 63, 187-191, 1990.

[19] Kohno, M., Yamada, M., Mitsuta, K., Mizuta, Y., and Yoshikawa, T.: Spin-trapping studies on the reaction of iron complexes with peroxides and the effects of water-solble antioxidants. Bull. Chem. Soc. Jpn., 64, 1447-1453, 1991.

[20] Hiramatsu, M., Kohno, M., Edamatsu, R., Mitsuta, K., and Mori, A.: Increased superoxide dismutase activity in aged human cerebrospinal fluid and rat brain determined by electron spin resonance spectrometry using the spin trap method. J. Neurochem., 58, 1160-1164, 1992.

[21] Sato, K., Akaike, T., Kohno, M., Ando, M., and Maeda, H.: Hydroxyl radical production by $\mathrm{H}_{2} \mathrm{O}_{2}$ plus $\mathrm{Cu}, \mathrm{Zn}$-superoxide dismutase reflects the activity of free copper released from the oxidatively damaged enzyme. J. Biol. Chem., 267, 25371-25377, 1992.

[22] Kohno, M., Mizuta, Y., Kusai-Yamada, M., Masumizu, T., and Makino, K.: Measurements of superoxide anion-radical and superoxide anion scavenging activity by electron-spinresonance spectroscopy coupled with DMPO spin-trapping. Bull. Chem. Soc. Jpn., 67, 1085-1090, 1994.

[23] Ogawa, N., Tanaka, K., Asanuma, M., Kawai, M., Masumizu, T., Kohno, M., and Mori, A.: Bromocriptine protects mice against 6-hydroxydopamine and scavenges hydroxyl free radicals in vitro. Brain Res., 657, 207-213, 1994.

[24] Noda, Y., Anzai, K., Mori, A., Kohno, M., Shinmei, M., and Packer, L.: Hydroxyl and superoxide anion radical scavenging activities of natural source antioxidants using the computerized JES-FR30 ESR spectrometer system. Biochem. Mol. Biol. Int., 42, 35-44, 1997.

[25] Unno, T., Sakane, I., Masumizu, T., Kohno, M., and Kakuda, T.: Antioxidative activity of water extracts of lagerstroemia speciosa leaves. Biosci. Biotech. Biochem., 61, 1772-1774, 1997.

[26] Saito, K., Kohno, M., Yoshizaki, F., and Niwano, Y.: Antioxidant properties of herbal extracts selected from screening for potent scavenging activity against superoxide anions. J. Sci. Food Agric., 88, 2707-2712, 2008.

[27] Saito, K., Kohno, M., Yoshizaki, F., and Niwano, Y.: Extensive screening for edible herbal extracts with potent scavenging activity against superoxide anions. Plant Foods Hum. Nutr., 63, 65-70, 2008.

[28] Saito, K., Takahashi, M., Ozawa, T., and Kohno, M.: Measurement of superoxide anion generated from oral polymorphonuclear leukocyte using novel spin trap, 5(diphenylphosphinoyl)-5-methyl-4,5-dihydro-3H-pyrrole Noxide (DPPMPO). Free Radic. Biol. Med., 43, S179, 2007.

[29] Nishizawa, M., Shioji, K., Kuraushi, Y., Okuma, K., and Kohno, M.: Spin-trapping properties of 5-(Diphenylphosphinoyl)-5-methyl-4,5-dihydro-3H-pyrrole $\mathrm{N}$-Oxide (DPPMDPO). Bull. Chem. Soc. Jpn., 80, 495-497, 2007.

[30] Finkelstein, E., Rosen, G.M., Rauckman, E.J., and Paxton, J.: Spin trapping of superoxide. Mol. Pharmacol., 16, 676-685, 1979.

[31] Finkelstein, E., Rosen, G.M., and Rauckman, E.J.: Spin trapping of superoxide and hydroxyl radical: practical aspects. Arch. Biochem. Biophys., 200, 1-16, 1980. 
[32] Miyazawa, H., Yoshikawa, T., Tanigawa, M., Yoshida, N., Sugino, S., Kondo, M., Nishikawa, M., and Kohno, M.: Measurement of serum superoxide-dismutase activity by electron-spin resonance. J. Clin. Biochem. Nutr., 5, 1-7, 1988.

[33] Yamazaki, I. and Piette, L.H.: ESR spin-trapping studies on the reaction of $\mathrm{Fe}^{2+}$ ions with $\mathrm{H}_{2} \mathrm{O}_{2}$-reactive species in oxygen toxicity in biology. J. Biol. Chem., 265, 1358913594, 1990.

[34] Makino, K., Hagi, A., Ide, H., Murakami, A., and Nishi, M.: Mechanistic studies on the formation of aminoxyl radicals from 5,5-dimethyl-1-pyrroline- $N$-oxide in fenton systemscharacterization of key precursors giving rise to background ESR signals. Can. J. Chem., 70, 2818-2827, 1992.

[35] Sato, E., Kato, M., Kohno, M., and Niwano, Y.: Clindamycin phosphate scavenges hydroxyl radical. Int. J. Dermatol., 46, 1185-1187, 2007.

[36] Sato, E., Mokudai, T., Niwano, Y., Kamibayashi, M., and Kohno, M.: Existence of a new reactive intermediate oxygen species in hypoxanthine and xanthine oxidase reaction. Chem. Pharm. Bull., 56, 1194-1197, 2008.

[37] Kohno, M., Sato, E., Yaekashiwa, N., Mokudai, T., and Niwano, Y.: Proposed mechanisms for $\mathrm{HOOOH}$ formation in two typical enzyme reactions responsible for superoxide anion production in biological systems. Chem. Lett., 38, 302307, 2009.

[38] Shibuki, K.: An electrochemical microprobe for detecting nitric oxide release in brain tissue. Neurosci. Res., 9, 69-76, 1990.

[39] Zafiriou, O.C., Mcfiriou, M.M., and Bromund, R.H.: Nitric oxide in seawater. Science, 207, 637-639, 1980.
[40] Kohno, M., Masumizu, T., and Mori, A.: ESR demonstration of nitric oxide production from nitroglycerin and sodium nitrite in the blood of rats. Free Radic. Biol. Med., 18, 451457, 1995.

[41] Hotta, Y., Otsuka-Murakami, H., Fujita, M., Nakagawa, J., Yajima, M., Liu, W., Ishikawa, N., Kawai, N., Masumizu, T., and Kohno, M.: Protective role of nitric oxide synthase against ischemia-reperfusion injury in guinea pig myocardial mitochondria. Eur. J. Pharmacol., 380, 37-48, 1999.

[42] Saito, K. and Kohno, M.: Application of electron spin resonance spin-trapping technique for evaluation of substrates and inhibitors of nitric oxide synthase. Anal. Biochem., 349, 16-24, 2006.

[43] Kosaka, H., Imaizumi, K., Imai, K., and Tyuma, I.: Stoichiometry of the reaction of oxyhemoglobin with nitrite. Biochim. Biophys. Acta., 581, 184-188, 1979.

[44] Tsuchiya, K., Takayanagi, M., Minakuchi, K., and Fukuzawa, K.: Sensitive quantitation of nitric oxide by EPR spectroscopy. Free Radic. Biol. Med., 21, 733-737, 1996.

[45] Anbar, M. and Neta, P.: A compilation of specific bimolecular rate constants for the reactions of hydrated electrons, hydrogen atoms and hydroxyl radicals with inorganic and organic compounds in aqueous solution. Int. J. Appl. Radiat. Isot., 18, 493-523, 1967.

[46] Beckman, J.S. and Koppenol, W.H.: Nitric oxide, superoxide, and peroxynitrite: the good, the bad, and the ugly. Am. J. Physiol. Cell Physiol., 271, C1424-C1437, 1996.

[47] Yaekashiwa, N., Sato, E., Niwano, Y., and Kohno, M.: Reactive oxygen species produced by human oral neutrophils belongs to free radical species. Free Radic. Biol. Med., 47 (Suppl. 1), S134, 2009. 